\title{
Intravitreal Ranibizumab for the Treatment of Choroidal Neovascularization in Best's Vitelliform Macular Dystrophy
}

\author{
Figen Batioglu ${ }^{\mathrm{a}}$, Emin Ozmert ${ }^{\mathrm{a}}$, Elcin Suren ${ }^{\mathrm{a}}$, Sibel Demirel ${ }^{\mathrm{a}, \mathrm{b}}$
}

\begin{abstract}
To evaluate the results of intravitreal ranibizumab injection in a case with choroidal neovascularization (CNV) in Best's vitelliform macular dystrophy with different imaging modalities. A 20 -year-old male with the complaint of reduced vision in the right eye underwent complete ophthalmological examination including fluorescein angiography (FA), fundus autofluorescence (FAF) imaging. Macular scans were obtained with spectral optical coherence tomography (OCT). Best corrected visual acuity was counting fingers at 2 meters in the right eye. Fluorescein angiography and OCT confirmed the diagnosis of CNV associated with Best's vitelliform macular dystrophy. After 3 monthly injections of intravitreal ranibizumab, $\mathrm{CNV}$ regressed and macular edema disappeared. Visual acuity improved to $10 / 10$. His condition remained stable for 3 years after treatment. We concluded that the intravitreal ranibizumab may be a new approach for the therapy of CNV in Best's vitelliform macular dystrophy. However, a long-term follow-up is warranted. Different imaging modalities help us to understand the different aspects of macular involvement in this disease complicated with CNV.
\end{abstract}

Keywords: Best's vitelliform macular dystrophy; Fundus autofluorescence; Intravitreal ranibizumab; Optical coherence tomography

\section{Introduction}

Best vitelliform macular dystrophy is an autosomal dominant disease caused by mutation in the gene coding for

\footnotetext{
Manuscript accepted for publication March 13, 2012

${ }^{a}$ Ankara University Faculty of Medicine, Department of Ophthalmology, Ankara, Turkey

${ }^{\mathrm{b} C o r r e s p o n d i n g ~ a u t h o r: ~ S i b e l ~ D e m i r e l, ~ K o z a ~ s o k . ~ M e s a ~ I ̇ k i z l e r ~ S i t e s i ~}$

70/40, G.O.P, Ankara, Turkey. Email: drsibeldemireltr@yahoo.com.tr

doi:10.4021/jmc619w
}

bestrophin [1]. It is characterized initially by symmetrical, bilateral, yellow-orange vitelliform lesions which gradually disrupt and progress to scarring. Usually, the visual prognosis for patients with Best's vitelliform macular dystrophy is good [2]. Also even in the presence of a large vitelliform lesion, patients typically retained relatively good visual acuity (VA). However, during the disruptive phase, subretinal hemarrhage may occur at the posterior pole in isolation or occur in association with choroidal neovascularization which may lead to a rapid decrease in visual acuity $[3,4]$. There are no evidence-based treatment options for CNV available to date. Photodynamic therapy [3], combined photodynamic therapy and intravitreal triamcinolone injection [4], argon laser photocoagulation [5], and intravitreal bevacizumab injection $[6,7]$ have been reported to be an effective treatment option in case reports.

We report a rare case of CNV secondary to Best's vitelliform macular dystrophy treated with intravitreal injection of ranibizumab and evaluated with different imaging modalities.

\section{Case Report}

A 20-year-old male with Best's vitelliform macular dystrophy complained of decreased vision in the right eye for 1 week. He was evaluated by complete ophthalmic examination, FA, FAF and spectral OCT. Best corrected visual acuity was counting fingers at 2 meters in the right eye, and 10/10 in the left eye. Biomicroscopic examination of the anterior segment was unremarkable in both eyes. Fundus examination revealed macular edema and hemorrhage in the right eye (Fig. 1a), and vitelliruptive stage of Best's disease in the left eye.

Fluoresecein angiography revealed early hyperfluorescence with late leakage confirmatory of CNV in the right eye along with hypofluorescence due to hemorrhage (Fig. 1b). Fundus autofluoresence image revealed hypoautofluoresence due to hemorrhage and CNV (Fig. 1c). Optical coherence tomography demonstrated hyperreflective mass protruding from the retinal pigment epithelium, corresponding to the $\mathrm{CNV}$ associated with subretinal fluid in the submacu- 

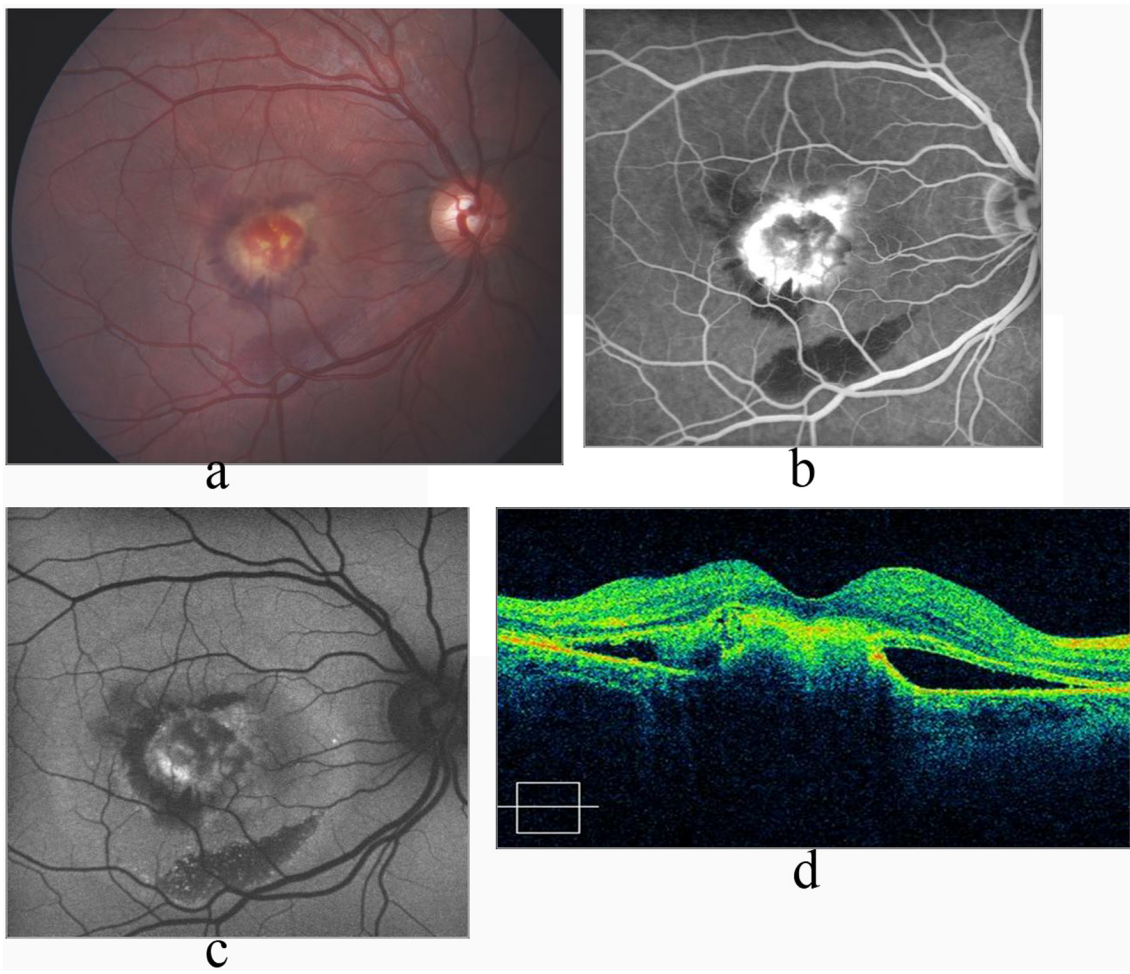

Figure 1. a: Macular edema and hemorrhage in the right eye in Best's disease; b: FA shows increased hyperfluoresence due to CNV, and hypofluorescence due to hemorrhage in the late phase; C: FAF image shows hypoautofluorescence due to subretinal classical CNV, and hemorrhage; d: OCT shows hyperreflective submacular CNV with subretinal fluid 6 months after the last injection of intravitreal ranibizumsb.

lar area (Fig. 1d). The study was conducted in compliance with the institutional and government review board regulations, informed consent regulations and the Declaration of Helsinki. After obtaining informed consent, 3 doses of intravitreal ranibizumab was performed by monthly intervals. Best corrected visual acuity had improved to $3 / 10$ and $10 / 10$ at first and 6th month of the last injection respectively. Fundus examination showed resolution of the subretinal fluid and hemorrhage and a scarred CNV (Fig. 2a). Fluorescein angiography disclosed hyperfluorescence due to staining of the regressed CNV (Fig. 2b) whereas FAF image showed hypoautofluorescence (Fig. 2c). OCT revealed significant reduction of the subretinal fluid with dome-shaped elevation of the outer hyperreflective retinal layer (Fig. 2d). During follow-up of 3 years, we noted neither recurrence of the CNV nor any drug-related ocular or systemic side effects. Visual acuity was 10/10.

\section{Discussion}

In Best's vitelliform macular dystrophy, usually a gradual loss of visual acuity occurs over a period of years. During the disruptive phase, subretinal hemorrhage may occur at the posterior pole. Many of these patients may spontaneously recover vision, depending on the amount of hemorrhage and degree of vision loss. Furthermore hemorrhage may be an indication in association with exudative manifestations secondary to $\mathrm{CNV}$ development which represents a major vision threatening complication. Fortunately $\mathrm{CNV}$ is a rare complication in Best's vitelliform macular dystrophy [2-5]. A neovascular membrane may be shown by FA and OCT. Optical coherence tomography demonstrate a high reflective lesion under the retinal pigmented epithelium associated with serous retinal detachment and may confirm the diagnosis [5].

Although histopathologic data is not clear, based on the results of treatment in the articles, it may be hypothesized that vascular endothelial growth factor (VEGF) may play a role in the pathogenesis of CNV secondary to Best's vitelliform macular dystrophy [3-7]. Intraviteral bevacizumab for the blockage of VEGF was reported in Best's disease and favorable results were acquired $[6,7]$.

Ranibizumab is a recombinant, humanized, monoclonal antibody antigen-binding fragment $(\mathrm{Fab})$ that neutralizes all biologically active forms of VEGF, effectively used in the treatment of neovascular age related macular degeneration [8]. Intravitreal ranibizumab injection was performed in two cases with Best's vitelliform macular dystrophy complicated with CNV $[9,10]$. In these case reports, one injection of ranibizumab induced total regression of $\mathrm{CNV}$ as early as by the 1-month follow-up In our patient, 3 doses of intravitreal ranibizumab injection by monthly intervals provided recov- 


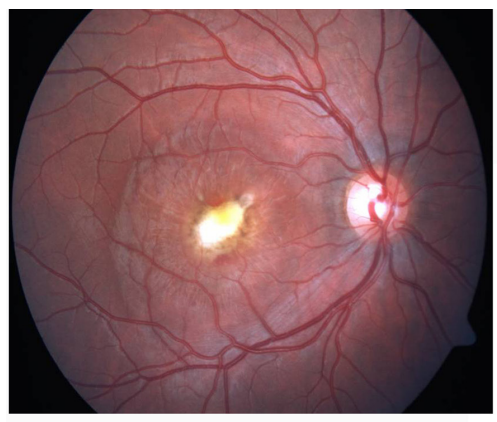

a

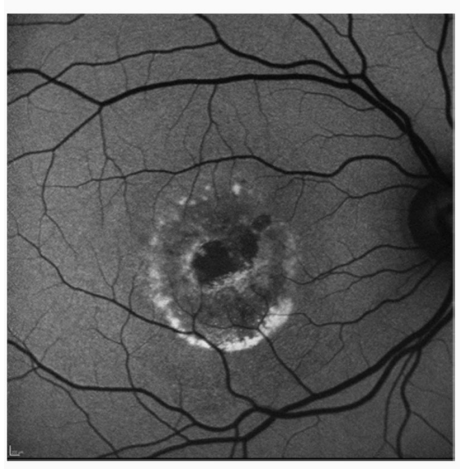

c

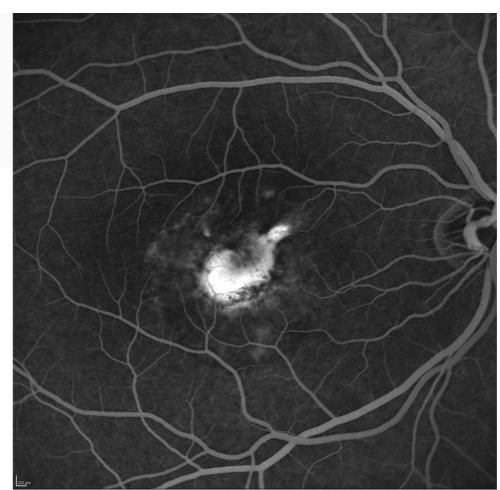

b

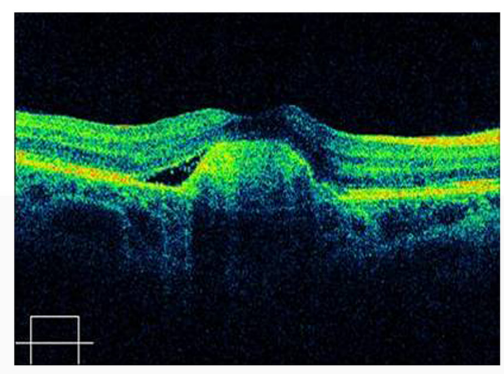

d

Figure 2. a: Color fundus photograph shows scarred CNV with resolution of the subretinal fluid and hemorrhage; b: FA shows absence of leakage and staining of the CNV; c: FAF image shows hypoautofluorescent scar with hyperautofluorescent deposits arround it; $d$ : Significant reduction of the subretinal fluid and retinal thickness on OCT.

ery of VA, regression of the $\mathrm{CNV}$, and reduction of macular edema on OCT.

In conclusion, intravitreal ranibizumab as an anti VEGF agent may be a new approach for the treatment of CNV in Best's vitelliform macular dystrophy. However, further studies are required to assess its efficiency and confidence for treatment. Different imaging modalities help us to understand the different aspects of macular involvement in this disease complicated with $\mathrm{CNV}$.

\section{Acknowledgement}

None of the authors have any proprietary interest. None of the authors have any financial support.

\section{References}

1. Allikmets R, Singh N, Sun H, Shroyer NF, Hutchinson A, Chidambaram A, Gerrard B, et al. A photoreceptor cell-specific ATP-binding transporter gene (ABCR) is mutated in recessive Stargardt macular dystrophy. Nat Genet. 1997;15(3):236-246.

2. Mohler CW, Fine SL. Long-term evaluation of patients with Best's vitelliform dystrophy. Ophthalmology.
1981;88(7):688-692.

3. Andrade RE, Farah ME, Costa RA. Photodynamic therapy with verteporfin for subfoveal choroidal neovascularization in best disease. Am J Ophthalmol. 2003;136(6):1179-1181.

4. Nobrega M, Bortolotto C, Farah M. Combined photodynamic therapy and intravitreal triamcinolone injection for choroidal neovascularization in Best disease. Can J Ophthalmol. 2007;42(5):761-762.

5. Andrade RE, Farah ME, Cardillo JA, Hofling-Lima AL, Uno F, Costa RA. Optical coherence tomography in choroidal neovascular membrane associated with Best's vitelliform dystrophy. Acta Ophthalmol Scand. 2002;80(2):216-218.

6. Leu J, Schrage NF, Degenring RF. Choroidal neovascularisation secondary to Best's disease in a 13-year-old boy treated by intravitreal bevacizumab. Graefes Arch Clin Exp Ophthalmol. 2007;245(11):1723-1725.

7. Rishi E, Rishi $P$, Mahajan S. Intravitreal bevacizumab for choroidal neovascular membrane associated with Best's vitelliform dystrophy. Indian J Ophthalmol. 2010;58(2):160-162.

8. Schmidt-Erfurth U. Clinical safety of ranibizumab in age-related macular degeneration. Expert Opin Drug Saf. 2010;9(1):149-165.

9. Querques G, Bocco MC, Soubrane G, Souied EH. In- 
travitreal ranibizumab (Lucentis) for choroidal neovascularization associated with vitelliform macular dystrophy. Acta Ophthalmol. 2008;86(6):694-695.

10. Heidary F, Hitam WH, Ngah NF, George TM, Hashim
H, Shatriah I. Intravitreal Ranibizumab for Choroidal Neovascularization in Best's Vitelliform Macular Dystrophy in a 6-Year-Old Boy. J Pediatr Ophthalmol Strabismus. 2011;48 Online:e19-22. 